\title{
Clinical Aspects, Microbiological Features, and Therapeutic Outcome of Brain Abscess: An Experience of a Referral Center
}

llad Alavi Darazam ( $\sim$ ilad13@yahoo.com )

Shahid Beheshti University of Medical Sciences

Firouze Hatami

Shahid Beheshti University of Medical Sciences

Shervin Shokouhi

Shahid Beheshti University of Medical Sciences

Masoud Mardani

Shahid Beheshti University of Medical Sciences

Minoosh Shabani

Shahid Beheshti University of Medical Sciences

Neda Khabiri

Shahid Beheshti University of Medical Sciences

Omidvar Rezaei

Shahid Beheshti University of Medical Sciences

Farid Javandoust Gharehbagh

Shahid Beheshti University of Medical Sciences

Mohammad Samadian

Shahid Beheshti University of Medical Sciences

Guive Sharifi

Shahid Beheshti University of Medical Sciences

Kaveh Ebrahimzadeh

Shahid Beheshti University of Medical Sciences

Seyed Ali Mousavinejad

Shahid Beheshti University of Medical Sciences

Mohammad Hallajnejad

Shahid Beheshti University of Medical Sciences

Mohammad Mahdi Rabiei

Shahid Beheshti University of Medical Sciences

Farahnaz Bidari

Shahid Beheshti University of Medical Sciences

Elena Jamali 
Shahid Beheshti University of Medical Sciences

\section{Shahram Sabeti}

Shahid Beheshti University of Medical Sciences

\section{Research Article}

Keywords: Brain abscess, Diagnosis, Therapy, Prognosis

Posted Date: March 7th, 2022

DOl: https://doi.org/10.21203/rs.3.rs-1417161/v1

License: (c) (1) This work is licensed under a Creative Commons Attribution 4.0 International License. Read Full License 


\section{Abstract \\ Background}

Brain abscess (BA) is recognized as a potentially fatal neurological infection requiring appropriate and prompt treatment. Improvements in diagnostic imaging and patient care have recently changed clinical symptoms, epidemiology, and outcomes. Therefore, we aimed to review all BA patients in our referral center.

\section{Method}

This study retrospectively reviewed all confirmed cases of BA referred to an academic teaching hospital and tertiary referral center between 2009 and 2021.

\section{Result}

The study included 119 clinically and radiologically confirmed brain abscesses (84\% male vs. $16 \%$ female) with a mean age of $38.6 \pm 16$ years (range:1-79 years). The cause of BA in most cases $(71.4 \%)$ was unknown. Only 11 patients had positive smears. Approximately $42.9 \%$ of patients were treated with surgery and antibiotic therapy, and the rest were treated with antibiotic therapy. The mortality rate in our study was $31.1 \%$. Based on our analysis, HIV patients (P: 0.003), IV drug users (P: 0.025), and patients with loss of consciousness at the time of admission $(P<0.001)$ had a high mortality rate. On the other hand, patients treated with surgical intervention exhibited a low mortality rate (P: 0.02). The most common site of BA in our patients was the parietal (27.2\%).

\section{Conclusion}

In our experience, HIV patients, IV drug users, and unconscious people on admission had higher mortality rates. The mortality rate of patients who underwent surgery was also low. The source of BA in most patients was unknown.

\section{Introduction}

Pyogenic brain abscess (BA) is a focal intracranial infectious complication that results from a variety of infections, surgery, and trauma [1]. Like abscesses elsewhere, BA begins primarily as a localized area of cerebritis and eventually transforms into an encapsulated collection of pus that resembles a mass-like lesion. [2].

Globally, BA is still a serious problem and accounts for about $8 \%$ of intracranial mass lesions [3]. In developing countries, the prevalence of BA is high due to poverty, poor sanitation, and delays in treatment 
[4]. Despite recent advances in neuroimaging techniques, antibiotic use, and surgical approaches, BA is recognized as a potentially fatal neurological infection requiring appropriate and prompt treatment [5]. The BA mortality rate is estimated to be between $8 \%$ and $25 \%$ in large case series $[6,7]$.

Clinical presentation of intracranial abscess depends on the location, size, number of lesions, brain structure involved, underlying medical conditions, and any secondary cerebral injury $[8,9]$. The clinical manifestation of BA can present in syndromes like focal mass expansion, intracranial hypertension, diffuse destruction, and focal neurological deficit [3].

A wide range of pathogens can lead to $B A$, depending on the patient's immune system and the development of the BA. However, overall, streptococci (both aerobic and anaerobic) are the most common pathogens [10].

Treatment of BA requires multidisciplinary approaches including surgery, the use of antibiotic therapy, and the eradication of primary infected foci [11]. Improvements in diagnostic imaging and patient care have recently changed clinical symptoms, epidemiology, and outcomes. Therefore, we aimed to review all BA patients in our referral center during the 2009-2021 in this study.

\section{Method}

This study retrospectively reviewed all confirmed cases of BA referred to an academic teaching hospital and tertiary referral center between 2009 and 2021. The electronic data in our hospital have been registered for the past 13 years. The diagnosis of BA had been confirmed by clinical manifestations (i.e., loss of cautiousness, neurological deficits) and imaging modalities such as brain computerized tomography (CT) scan and/or brain magnetic resonance imaging (MRI).

BA sources were based on known common risk factors such as congenital heart disease, ear, nose, and throat infection and surgery, lung abscess and bronchiectasis, head trauma, neurosurgery, dental infection, organ transplant, immunomodulatory therapies, cancer and connective tissue disease.

Demographic and clinical data including age, gender, past medical history, sign and symptoms at admission, laboratory data, imaging results, the duration of treatment during hospitalization, the therapeutic regimen, and early outcome (recovery upon discharge and mortality rate) were extracted.

Results of biopsy and pus from brain abscesses that had been collected during neurosurgery by craniotomy were extracted. The association between mortality and some variable, including diabetes mellitus (DM), sex, congenital heart failure, HIV, symptoms, the surgical intervention, the location of brain abscess, probable source, smear, and the medical regimen treatment was investigated by chi-square test.

The present study was approved by the Research Ethics Committee IR.SBMU.RETECH.REC.1400.052.

\section{Result}


The study included 119 clinically and radiologically confirmed brain abscesses (84 males vs. 16 females) with a mean age of $38.6 \pm 16$ years (range: $1-79$ years). The possible sources of BA varied in this study. The cause of BA in most cases $(71.4 \%)$ was unknown. Other sources for BA were previous head trauma $(6.7 \%)$, chronic sinusitis $(5.9 \%)$, iatrogenic $(5.9 \%)$, mastoiditis $(3.4 \%)$, shunt congenital heart disease (2.5\%), media otitis (2.5\%), and endocarditis (1.7\%). As outlined in Table 1, patients showed clinical symptoms such as headache (66.4\%), fever (53.8\%) and loss of cautiousness $(25.2 \%)$.

Table 1

Clinical sign and symptoms

\begin{tabular}{|lll|}
\hline Sign and symptoms & $\mathbf{N}$ & $\%$ \\
\hline headache & 79 & $66.4 \%$ \\
\hline fever & 64 & $53.8 \%$ \\
\hline Loss of consciousness & 39 & $32.8 \%$ \\
\hline Nausea and vomiting & 30 & $25.2 \%$ \\
\hline seizure & 29 & $24.4 \%$ \\
\hline dizziness & 20 & $16.8 \%$ \\
\hline paresis & 19 & $16 \%$ \\
\hline Gait ataxia & 13 & $10.9 \%$ \\
\hline paresthesia & 12 & $10.1 \%$ \\
\hline diplopia & 12 & $10.1 \%$ \\
\hline Blurred vision & 8 & $6.7 \%$ \\
\hline
\end{tabular}

Of the 51 patients who underwent surgery, 30 had a positive biopsy for a brain abscess. Eleven patients had positive smears and positive cultures (2.5\% coagulase-negative Staphylococci, $1.7 \%$ Viridans streptococci, 1.7\% Klebsiella, 0.8\% Pseudomonas, 0.8 E.coli, 0.8\% Staphylococci aureus, and 0.8\% Aspergillus). In this study, about $9.4 \%$ of unknown cases were culture positive. Approximately $42.9 \%$ of patients were treated with surgery and antibiotic therapy, and the rest were treated with antibiotic therapy. The location of the brain abscess varied among the patients. The most common site in our patients was the parietal (27.2\%) (Table 2). 
Table 2

Location of BA

\begin{tabular}{|lll|}
\hline Location & N & $\%$ \\
\hline Parietal & 33 & $27.7 \%$ \\
\hline Temporal & 21 & $17.6 \%$ \\
\hline Frontal & 15 & $12.6 \%$ \\
\hline Parietotemporal & 12 & $10.0 \%$ \\
\hline Multiple & 12 & $10.0 \%$ \\
\hline Frontoparietal & 7 & $5.8 \%$ \\
\hline Parietooccipital & 6 & $5.0 \%$ \\
\hline Occipital & 5 & $4.2 \%$ \\
\hline Cerebellum & 4 & $3.3 \%$ \\
\hline Frontotemporal & 4 & $3.3 \%$ \\
\hline
\end{tabular}

The treatment duration during hospitalization was different among the patients (mean: 24.6, SD: 16.1 day). The antibiotic regimens used for treatment were ceftriaxone plus metronidazole and vancomycin (78.2\%), ceftriaxone plus metronidazole plus vancomycin and cotrimoxazol (6.7\%), metronidazole plus vancomycin $(5.9 \%)$, voriconazole $(1.7 \%)$, ceftriaxone plus meropenem and vancomycin $(0.8 \%)$, ceftriaxone plus metronidazole plus vancomycin and amikacin $(0.8 \%)$, ceftazidim plus metronidazole and vancomycin $(0.8 \%)$, metronidazole plus vancomycin and amphotericin $(0.8 \%)$.

The mortality rate in our study was $31.1 \%$. Based on our analysis, HIV patients (P: 0.003$)$, IV drug users (P: 0.025), and patients with loss of consciousness at the time of admission $(P<0.001)$ had a high mortality rate. On the other hand, patients treated with surgical intervention exhibited a low mortality rate (P: 0.02).

Some of our patients had other diseases, including HIV (23.5\%), IV drug users (20.2\%), hepatitis C virus ( $\mathrm{HCV})(7.6 \%), \mathrm{DM}(7.6 \%)$, congenital heart failure (5\%), transplant organ (3.4\%) and rheumatoid arthritis $(1.7 \%)$.

\section{Discussion}

In this study, the source of BA in most cases was unknown, and the majority of patients were diagnosed with HIV. A case-control study investigating and quantifying potential risk factors for the BA showed that the odds ratio for HIV was 12.08 (95\% $\mathrm{Cl} 6.13-23.7)$. Based on this study, HIV or immunomodulatory therapies, including cytotoxic agents and biological treatments were important risk factors for the BA [12]. 
Another study showed that the BA was more often seen in immunocompromised patients [13]. BA should be considered a severe fatal infection, as increased opportunistic infections can increase the likelihood of BA and death in immunocompromised patients such as HIV-infected patients and transplant recipients. $[14,15]$. Because immunodeficiency is an important risk factor for BA; therefore, diagnostic work-ups for HIV in brain abscess patients could be helpful.

In another study, 75 patients were tested over a 5-year period. The main factors that increased mortality from intracranial abscesses were age, delayed hospitalization, focal neurological impairment, patient immunodeficiency, and a Glasgow coma scale (GCS) of less than 12 [16]. Consistent with this study, our study found a link between mortality with immunodeficiency status, and low GCS, while, there was no association between age and other symptoms. In their study and ours, there was no association between mortality and culture results or the number of abscesses.

In another study, patients with good GCS on admission, no underlying disease, positive cultures, or surgical treatment were more likely to have good outcomes. However, patients with acquired immunodeficiency syndrome, hematologic disease, and medical treatment alone exhibited poor outcomes [17]. In agreement with this study, our study showed that the mortality rate was lower in patients who were treated with surgical intervention and there was no association between mortality and result of culture.

Due to the unknown sources of most BA cases, the treatment is a major challenge $[4,13]$. A retrospective epidemiological study showed that the majority of BA cases with unknown origins were culture-negative [18]. In our study, a few numbers of BA cases with unknown sources were culture-positive, suggesting that cultures' results carried low sensitivity for BA diagnosis and were not helpful.

The proportion of positive bacterial cultures in another series was 12\% [19]. Administering antibiotics before collecting abscess material could explain sterile cultures. Because BA is a life-threatening condition, empirical treatment is usually initiated before the results of the culture are available [20].

BA is a fatal disease and initial antibiotic therapy is a necessity based on suspected sources. Anaerobic pathogens should be considered by administrating of the empirical antibiotics, such as a third-generation cephalosporin and metronidazole plus vancomycin. Other antibiotics can be added depending on the predisposition and the outcome of the pus culture $[11,21]$. If the culture is negative, broad-spectrum antibiotics should be continued [22]. Ceftriaxone and metronidazole and vancomycin were most commonly used in our study because most patients had negative cultures and the cause of most cases was unknown.

The antibiotics used in BA are based on suspicious sources, and if unknown, empirical antibacterial treatments are based on common sources. In this study, there was no evidence of imaging and physical exam for common source; therefore, routine antibacterial treatment had been modified in some unknown cases. 
In our study, the duration of treatment during hospitalization varied among the patients. The duration of BA antibiotic therapy should be personalized based on the size of the BA, response to treatment, and the need for surgical intervention [4].

In a review, mortality ranged from 5-32\% (4). In our study, the mortality rate was about $31 \%$. The high mortality rate may be due to the high prevalence of HIV-infected patients in our study. There were some limitations in our study. Since our study was a retrospective study, it was dependent on physicians' judgments. There were also no objective measures of health and functional limitations were observed in the follow-up of patients.

\section{Conclusion}

In our experience, HIV patients, IV drug users, and unconscious people on admission had higher mortality rates. The mortality rate of patients who underwent surgery was low. The source of BA in most patients was unknown. The results of BA culture in most patients were negative.

\section{Declarations}

Ethics approval: The present study was approved by the Research Ethics Committee IR.SBMU.RETECH.REC.1400.052.

Consent for publication: Not applicable.

Consent for participant: Not applicable.

Availability of data and materials: The datasets generated during and/or analysed during the current study are available from the corresponding author on reasonable request.

Competing insert: We declare no competing interests

Funding: Not applicable

Authors' contributions: All authors contributed to conception and design of study; FH, IAD, NKH contributed to the acquisition of data; FH, IAD contributed to the analysis of data; all authors contributed to the drafting of the article and/or critical revision; and all authors contributed to the final approval of manuscript.

Acknowledgments: The authors would like to thank the Clinical Research Development Unit (CRDU) of Loghman Hakim Hospital, Shahid Beheshti University of Medical Sciences, Tehran, Iran for their help and support in conducting this study.

\section{References}


1. Nathoo N, Nadvi SS, Narotam PK, van Dellen JR. Brain abscess: management and outcome analysis of a computed tomography era experience with 973 patients. World neurosurgery. 2011;75(56):716-26.

2. Wiwanitkit S, Wiwanitkit V. Pyogenic brain abscess in Thailand. North American journal of medical sciences. 2012;4(6):245.

3. Muzumdar D, Jhawar S, Goel A. Brain abscess: an overview. International journal of surgery. 2011;9(2):136-44.

4. Miranda HA, Leones SMC, Elzain MA, Moscote-Salazar LR. Brain abscess: current management. Journal of neurosciences in rural practice. 2013;4(S 01):S67-S81.

5. Kumar A, Saeed H, Alamri A, Crocker M, Dave J. Twenty years of intracranial abscesses: prognostic indicators and treatment review. Journal of Infection. 2011;63(6):491-2.

6. Gutiérrez-Cuadra M, Ballesteros M, Vallejo A, Miñambres E, Fariñas-Alvarez C, García-Palomo J, et al. Brain abscess in a third-level hospital: epidemiology and prognostic factors related to mortality. Revista espanola de quimioterapia: publicacion oficial de la Sociedad Espanola de Quimioterapia. 2009;22(4):201-6.

7. Helweg-Larsen J, Astradsson A, Richhall H, Erdal J, Laursen A, Brennum J. Pyogenic brain abscess, a 15 year survey. BMC infectious diseases. 2012;12(1):1-10.

8. Yang S-Y. Brain abscess: a review of 400 cases. Journal of neurosurgery. 1981;55(5):794-9.

9. Chun CH, Johnson JD, Hofstetter M, Raff MJ. Brain abscess. A study of 45 consecutive cases. Medicine. 1986;65(6):415-31.

10. Derber CJ, Troy SB. Head and neck emergencies: bacterial meningitis, encephalitis, brain abscess, upper airway obstruction, and jugular septic thrombophlebitis. Medical Clinics. 2012;96(6):1107-26.

11. Muzumdar D. Central nervous system infections and the neurosurgeon: a perspective. International journal of surgery (London, England). 2010;9(2):113-6.

12. Bodilsen J, Dalager-Pedersen $M$, van de Beek D, Brouwer MC, Nielsen $H$. Risk factors for brain abscess: A nationwide, population-based, nested case-control study. Clinical Infectious Diseases. 2020;71(4):1040-6.

13. Bodilsen J, Dalager-Pedersen $M$, van de Beek D, Brouwer M, Nielsen H. Incidence and mortality of brain abscess in Denmark: a nationwide population-based study. Clinical Microbiology and Infection. 2020;26(1):95-100.

14. Britt RH, Enzmann DR, Remington JS. Intracranial infection in cardiac transplant recipients. Annals of Neurology: Official Journal of the American Neurological Association and the Child Neurology Society. 1981;9(2):107-19.

15. Vieira J, Frank E, Spira TJ, Landesman SH. Acquired immune deficiency in Haitians: opportunistic infections in previously healthy Haitian immigrants. New England Journal of Medicine. 1983;308(3):125-9. 
16. Menon S, Bharadwaj R, Chowdhary A, Kaundinya D, Palande D. Current epidemiology of intracranial abscesses: a prospective 5 year study. Journal of medical microbiology. 2008;57(10):1259-68.

17. Xiao F, Tseng M-Y, Teng L-J, Tseng H-M, Tsai J-C. Brain abscess: clinical experience and analysis of prognostic factors. Surgical neurology. 2005;63(5):442-9.

18. LU CH, CHANG WN, LIN YC, TSAI NW, LILIANG PC, SU TM, et al. Bacterial brain abscess: microbiological features, epidemiological trends and therapeutic outcomes. Qjm. 2002;95(8):501-9.

19. Song L, Guo F, Zhang W, Sun H, Long J, Wang S, et al. Clinical features and outcome analysis of 90 cases with brain abscess in central China. Neurological Sciences. 2008;29(6):425-30.

20. Patel K, Clifford DB. Bacterial brain abscess. The Neurohospitalist. 2014;4(4):196-204.

21. Mace SE. Central nervous system infections as a cause of an altered mental status? What is the pathogen growing in your central nervous system? Emergency Medicine Clinics. 2010;28(3):535-70.

22. de LOUVOIS J. Bacteriological examination of pus from abscesses of the central nervous system. Journal of clinical pathology. 1980;33(1):66-71. 